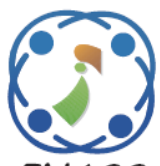

\title{
Load Balancing in RPL to Avoid Hotspot Problem for Improving Data Aggregation in IoT
}

\author{
Vanathi Arunachalam ${ }^{1 *}$ \\ Nagamalleswara Rao Nallamothu ${ }^{2}$ \\ ${ }^{1}$ Acharya Nagarjuna University, Aditya Engineering College, AP India \\ ${ }^{2} R V R \& J C$ College of Engineering, Guntur, AP, India \\ * Corresponding author's Email: vanathi_a@rediffmail.com
}

\begin{abstract}
Data aggregation plays a vital role in the Internet of Things (IoT), and it aggregates the collected sensor data from devices to suppress redundant data transmissions. Many-to-one traffic pattern in the IoT induces hotspot problem and inefficient data aggregation. The Routing protocol for low-power and lossy networks (RPL) in the network layer impacts the hotspot problem due to the frequent usage of forwarding nodes and load imbalance. The processes of network layer protocol, such as trickle algorithm and Objective Functions (OF) for Destination Oriented Directed Acyclic Graph (DODAG) construction, need more attention to avoid hotspot for efficient data aggregation. This work proposes a Load Balanced RPL (LoB-RPL) protocol to avoid hotspot creation using a composite metric based parent selection, DODAG construction, and local topology adaptive decision on trickle parameters. The LoBRPL improves the Minimum Hop with Hysteresis Objective Function(MRHOF) using the composite metric based parent selection and tunes the parameters of the Trickle algorithm. It ensures efficient maintenance of DODAG structure, hotspot avoidance, and unnecessary DIO transmissions. Beyond the advantages of composite metric based parent selection, consideration of dynamic parameters may induce frequent parent switching in RPL. To avoid frequent changes in the DODAG structure, the LoB-RPL optimally decides the parent switching threshold. Thus, the proposed work ensures a load-balanced and an energy-efficient RPL routing as well as data aggregation in the IoT environment. The LoB-RPL delivers outperforming results compared to the base RPL under various inter-packet interval time over 50 node topologies.
\end{abstract}

Keywords: Data aggregation, RPL, IoT, Hotspot problem, Load balancing, Energy efficient DODAG structure, Trickle algorithm, Hysteresis function.

\section{Introduction}

With the tremendous growth of the Internet of Things (IoT) applications, balanced utilization of resources among sensor devices becomes a crucial factor [1]. Data aggregation is a vital operation for improving the efficiency of IoT communication and network lifetime. Routing Protocol for Low Power and Lossy Networks (RPL) is a widely adopted protocol for IoT networks [2]. The functionalities of the network layer harm the aggregation efficiency. The RPL processes at the network layer may induce uneven energy dissipation among sensor nodes. The RPL constructs the Destination Oriented Directed Acyclic Graph (DODAG) to connect the sensors and root node with the support of different Objective Functions (OFs), and trickle algorithm [3-6]. As per RPL, heavy load is applied on the sensor nodes nearer to the root node than other, to route the datapackets to the root node. It induces the hot spot problem. Thus, improving the efficiency of RPL in the network layer is essential to utilize the advantages of data aggregation techniques in the application layer completely.

The RPL includes a built-in energy-saving mechanism in the DODAG construction process, such as the Trickle algorithm [7], which aims to minimize the number of route updates and message broadcast in the network while maintaining the DODAG structure. However, the Trickle algorithm mainly faces the issue of load imbalancing. The load 
imbalance among nodes, i.e., some of the nodes do not get a chance to broadcast the DIO messages. The conventional DODAG construction algorithms exclusively rely on a single routing metric, either hop count or Expected Transmission Count (ETX), for energy-efficient routing path to the sink node. The RPL routing with the hop count tends the nodes to uneven energy dissipation and hotspot issue. If it takes only ETX into account, time delay suffers the communication between nodes. There is no explicit mechanism to avoid hotspot creation due to load imbalance in the RPL protocol. Recently, several research works have designed the parent selection mechanism in RPL to avoid parent selection with the worst resource and link quality as well as avoiding a path with large hop count, workload, and Expected Transmission Count (ETX) [8]. However, these schemes tend to the phenomenon of repeated parent switching from one to the other. If repeated parent switching occurs on a node, it changes the parent node frequently and redesigns the DODAG structure continuously. To solve such issues, the proposed work plans to improve both the parent selection and trickle algorithm. The process of DODAG structure construction by selecting the parent node takes a composite metric of hop count, workload, and ETX in load-balanced DODAG structure. It can eliminate the issues of routing when considering a single routing metric. An uneven load distribution among nodes may enforce the nodes to frequently change the DODAG structure and worsen the network lifetime. The proposed work attempts to solve such issues by estimating the parameters in the trickle algorithm optimally and avoids frequent parent switching due to the consideration of composite metric in RPL. A node in Trickle believes that it selects an optimal DIO transmission interval and it believes that the randomly selected DIOs interval is sufficient to hear an enough number of DIOs transmissions. However, the randomly chosen DIOs interval time may fail to hear sufficient number of DIOs from neighboring nodes. To avoid such load imbalancing issue, the proposed methodology takes into account the successive DIOs suppressions and selects an optimal DIOs interval period. Thus, achieving a better load balance among neighboring nodes using the proposed routing technique paves to improve the data aggregation efficiency and IoT network lifetime.

The main contributions of the proposed methodology are as follows.

> The primary objective of the proposed algorithm is to support an energy-efficient data aggregation in the application layer by avoiding the hotspot problem using the improved network layer RPL activities.
Adopting improved MRHOF as the objective function in LoB-RPL to decide the number of candidate parents and maintain an energy-efficient DODAG structure with the minimized cost of routing overhead.

$>$ Deciding the count of consistent message transmission $\mathrm{k}$ with the knowledge of local-topology reduces the routing overhead without losing the DODAG efficiency.

$>$ Incorporating the successive DIOs suppression counter in the selection of the $t$ value of the Trickle algorithm avoids unnecessary comparisons between $\mathrm{k}$ and redundancy counter $\mathrm{c}$ and unnecessary DIO message traffic.

$>$ The improved trickle algorithm avoids an improper value of $\mathrm{c}$ due to the loss of consistentmessages and induces energy-efficient and reliable packet delivery.

$>$ A network stability based algorithm avoids frequent parent switching due to the consideration of composite routing metrics.

\subsection{Organization of the work}

Section 2 illustrates the existing routing techniques that improve the data aggregation efficiency as well as network lifetime. Section 3 formulates the routing problem associated with the data aggregation efficiency in IoT application layer. The details of the proposed methodology and its simulation results are given in section 4 and 5 respectively. Finally, section 6 concludes the work.

\section{Related works}

Data aggregation collects the data and aggregates it from the sensor's node to improve the network lifetime. Several data aggregation methods have been used in the application layer of IoT [9]. The existing data aggregation techniques based on clustering and region attempts to reduce duplicate data transmission across the network to aggregate the data among the network nodes, a suitable energy efficient routing protocol have to be used in the network layer. An energy and delay aware routing protocol [10] consists of two processes, namely parent selection and aggregation. The selection process takes into account Residual energy (RER) to select the optimal parent. The data aggregation process utilizes the theory of Compressed Sensing (CS). Then, the aggregated data is transmitted to the gateway through DODAG parent nodes. However, the protocols used in the lower layer need to support the data aggregation efficiency without degrading the network lifetime. A widely used protocol for IoT routing is RPL. Several works have been proposed to improve the performance in 
different concepts such as objective function and trickle algorithm [11] [12]. The improved RPL routing works exploit different routing metrics to make it dynamic and more adaptive to the IoT environment. However, the RPL has to be improved to solve load balancing and hotspot problem. Most of the conventional schemes exploit delay-based metrics in their objective function, such as hop count in the DODAG construction process. However, they enforce the nodes to select the paths through the distance routers, which are prematurely depleting their energy that leads to hotspot problems, and the energy of other nodes remains unused $[13,14]$. Some of the conventional schemes take into account the metric of ETX to minimize the number of transmissions or delay of packet routing to the DODAG root node. However, the consideration of ETX as a primary metric tends the network to uneven energy distribution among the nodes, causing poor network stability [15]. In paper [16], an energyefficient routing technique is proposed to show the importance of energy-efficient RPL routing in the IoT environment. It allows the nodes to take the RPL routing decision dynamically by considering the remaining energy of nodes and the energy required to route the data traffic. As per RPL routing, the node which is closer to the DODAG root node is involved in high traffic and depleted its energy completely. However, those routing metrics are not sufficient to meet the constraints of IoT, and it tends to serious load balancing problems in a network when heavy traffic networks are deployed with the RPL. The proposed Skewness and Balancing of RPL Trees for IoT networks (SB-RPL) has improved RPL with the combination of routing metrics [17]. The control overhead can be reduced further in such existing works by adjusting the RPL parameters. The RPL based on a flexible trickle algorithm in [18] considers fuzzy logic and provides a set of variables as input to enforce even energy dissipation without affecting the routing performance. However, there is a possibility for enhancing routing performance, using several networks and RPL parameters. Moreover, most of the conventional schemes lack in considering the network stability issue due to the consideration of network traffic related metrics, since those metrics are changed dynamically, and so the DODAG structure is hanged frequently.

The proposed Trickle-plus algorithm in [19] aims at minimizing energy consumption and improving the lifetime of the network. The trickle-plus algorithm considers three configuration parameters, such as shift factor, Ishift start, and Ishift end. The first parameter represents the amount of doubling intervals, and the last two parameters stand for the start and the end time of the Ishift. The usage of these parameters reduces the energy consumption of nodes as well as the delay of DODAG construction. However, the listen-only period results in load balancing and high routing delay issues. To handle this issue, new dynamic approaches are proposed [20]. The main idea behind those schemes is extending the listen-only period in conventional trickle algorithms to enable the node to listen to DIO message broadcasting for a long time. The elastic trickle timer algorithm is proposed in [21]. This trickle algorithm dynamically selects the listen-only period, according to the number of neighbors. However, the assumption of an even distribution of nodes in the network is not realistic. The trickle-I algorithm in [22] enables the nodes to hear the DIO message transmissions from the time $t$ to the end of the interval. For that, the Trickle I algorithm sets the value of redundancy counter as zero even during the DIO transmission as well as in suppression. It can reduce energy consumption as well as balance the network load among nodes. However, the random selection of $t$ value in the trickle algorithm reduces the routing performance.

\section{Problem formulation}

Internet of Things is modeled as a graph $(\mathrm{G})$, and the network $\mathrm{G}$ includes a set of sensors $(\mathrm{N})$, root node $(R)$, and edges (E). Moreover, $E=\{i, j\} \in N$ represents the set of direct connections between the nodes. During the network initialization, all nodes are installed with the same energy En. As per the RPL, the nodes send DIO message with the cost information, and they select a neighbor as a parent $(\mathrm{Np})$, which has minimum cost value. Finally, a full topology constructed as per RPL from each sensor to the root node is called as DODAG. The primary objective of this work is to improve the accuracy of data aggregation $\mathrm{D}(\mathrm{A})$ with the support of network layer protocol, RPL.

Factors Impacting Network Layer Protocol on the Efficiency of Data Aggregation: In the aspect of the network layer, the function of $\mathrm{D}(\mathrm{A})$ mainly depends on the number of generated $\left(\mathrm{DATA}_{\mathrm{G}}\right)$ data packets per node and delivered data packets from node $\mathrm{Ni}$ $\left(D A T A_{D}\right)_{N i}$ to a node $\mathrm{R}$, which performs aggregation. For example, if $\mathrm{D}(\mathrm{A})$ is average, the accuracy of $\mathrm{D}(\mathrm{A})$ is as follows, where, $|\mathrm{N}|$ represents a total number of nodes in the network.

$$
\begin{aligned}
& \text { Accuracy of } D(A)= \\
& \sum_{N i \in N}\left(D A T A_{D}\right)_{N i} /|N| \times D A T A_{G}
\end{aligned}
$$


There exists a root node $\mathrm{R}$ to which the data is to be aggregated. Exploring the literature, it can be understood that numerous efforts have been made to provide an energy-efficient DODAG either with hop count or ETX metric. They provide a minimum-cost DODAG for data aggregation. However, the accuracy of $\mathrm{D}(\mathrm{A})$ is affected.

The hop count and ETX based DODAG offer minimum latency and minimum ETX paths to the node $\mathrm{R}$, respectively. The relation between those metrics and accuracy of $\mathrm{D}(\mathrm{A})$ is as follows. Where, Bitdr represents the number of forwarded meaningless DATA due to the dropping of a particular number of bits at node Ni. When the factor of Hop Count, he increases, it harms the D(A) accuracy.

$$
\begin{gathered}
\text { Minimized Accuracy of } D(A) \text { due to } h c= \\
\left(\sum_{N i \in N}\left(D A T A_{D}\right)_{N i} /|N| \times D A T A_{G}\right)- \\
\left.\sum_{N i \in N}\left(\text { Bit } d r_{D}\right)_{N i} /|N| \times D A T A_{G}\right)
\end{gathered}
$$

Over a time, hc based DODAG construction in RPLworsen the problem of minimizedD(A) accuracy, due to the creation of hotspot around node R. Since, the hotspot problem increases the value of $\left(\sum_{\mathrm{Ni} \in \mathrm{N}}\right.$ (Bit $\left.\left.\mathrm{dr}_{\mathrm{D}}\right) \mathrm{N}_{\mathrm{i}}\left|\mathrm{N}_{\mathrm{i}}\right| \mathrm{xDATA}_{\mathrm{G}}\right)$. The consideration of ETX may increase the delayed packets, (Del $\left.\mathrm{fr}_{\mathrm{D}}\right)_{\mathrm{Ni}}$ and make them invalid at parent node, $\mathrm{Np}$ before data aggregation. It reduces the accuracy of $\mathrm{D}(\mathrm{A})$.

$$
\begin{gathered}
\text { Minimized Accuracy of } \mathrm{D}(\mathrm{A}) \text { due to ETX }= \\
\left(\sum_{\mathrm{Ni} \in \mathrm{N}}\left(\mathrm{DATA}_{\mathrm{D}}\right)_{\mathrm{Ni}} /|\mathrm{N}| \times \mathrm{DATA}_{\mathrm{G}}\right)- \\
\left(\sum_{N i \in N}\left(\operatorname{Del} f r_{D}\right)_{N i} /|N| \times D A T A_{G}\right)
\end{gathered}
$$

Another reason behind the problem is high overhead due to unnecessary DIO transmission. The basic trickle algorithm induces it in RPL. High overhead also leads to high $\left(B i t \mathrm{dr}_{\mathrm{D}}\right)$ Niand (Del $\left.\mathrm{fr}_{\mathrm{D}}\right)$ Ni. Thus, the proposed LoB-RPL routing protocol implements three processes, such as improved MRHOF using hc, ETX, and workload and local topology adaptive trickle algorithm. However, the consideration of workload may tend to cost dynamicity even after immediate to the parent changes in DODAG structure and network instability. Thus, the proposed work introduces a dynamic parent switching threshold factor in cost estimation.

\section{Overview of the proposed system}

The proposed methodology focuses on two important aspects of RPL protocol in the network layer, such as providing perfect parent selection and network stability by avoiding frequent parent switching to solve the impact of hotspot problems on the efficiency of data aggregation. The improved MRHOF considers a composite of routing metrics, such as hop count, workload, and ETX in preferred parent selection. The trickle plays an important role in deciding the efficiency of improved MRHOF. However, avoiding the hotspot problem through load balancing in RPL is successful, when dispersing a large amount of data traffic to different parents. Furthermore, without considering the sudden changes in DODAG structure due to frequent switches of the parent node, the proposed methodology lacks in attaining the communication efficiency and network stability always. Thus, the proposed method takes into account the metric of the parent switching threshold to avoid network instability. Fig. 1 shows the block diagram of LoBRPL.

\subsection{Preliminaries of RPL and its limitations related to hotspot problem}

The RPL consists of two main components, such as OF and Trickle algorithm. The RPL constructs the tree, called DODAG, and executes the many-to-one traffic using the components above. For that, the RPL includes three types of messages, such as

$>$ DODAG Information Objects (DIO) to build the DODAG and allow other nodes to discover an RPL instance and join it,

$>$ DODAG Information Solicitations (DIS) to solicit the DIO messages from other neighbor nodes,

$>$ Finally, DODAG Destination Advertisement Objects (DAO) ensures the downward paths to the parent node.

The primary function of RPL is to construct the DODAG structure using its MRHOF. However, most of the conventional MRHOFs exploit the hop count metric, and they allow RPL to determine stable minimum-latency paths from the nodes to a root node.

Some of the works use MRHOF with the ETX metric, and they enforce the RPL to select the stable minimum-ETX paths from the nodes to a rootnode. Both of them lack in considering the load level of nodes. Thus, it tends the sensor nodes nearer to the root node to die earlier and creates the hotspot around the root node. The proper usage of DIO messages using the trickle algorithm of RPLhas control over other RPL activities. Each node schedules DIO message transmission at a random time $\mathrm{t}$ in I interval. During the interval, the node keeps track of the number ofreceived messages using a redundancycounter $\mathrm{c}$. If $\mathrm{c}$ is below a redundancy threshold $\mathrm{k}$, the node transmits the DIO message; 


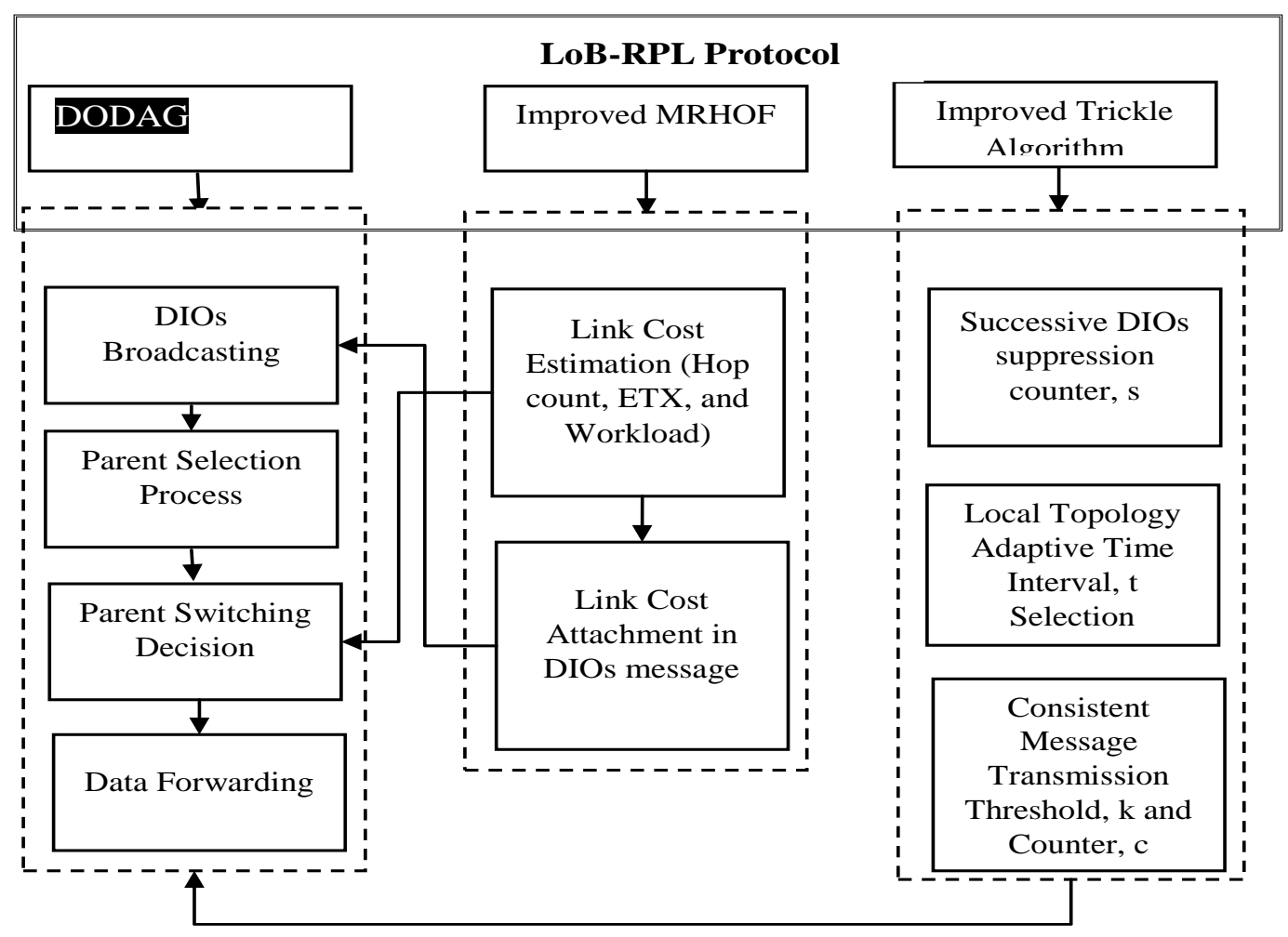

Figure. 1 Block diagram of the proposed methodology

otherwise, the transmission is suppressed. A main issue associated with the basic trickle algorithm is statically deciding trickle parameters. Thus, it is important to consider the decision of setting $\mathrm{k}$ value in the trickle algorithm, to avoid load imbalancing due to improper parent selection. Another important parameter in the Trickle algorithm is the selection of random time. Furthermore, the selected parent must be in the same status for at least a minimum amount of time; otherwise, the load-balanced RPL routing tends to poor performance. However, the consideration of traffic based routing metrics in parent selection may frequently change the cost of a node and induce repeated parent switching.

To avoid those issues in RPL, the proposed LoBRPL is proposed with the proper design of objective function and the Trickle algorithm.

\subsection{Adopting improved MRHOF as objective function in RPL}

The proposed algorithm exploits the load level to metric compute the link cost. If the RPL only considers the hop count, the nodes are suffered from uneven energy and hotspot issues. If it only takes into account the ETX, time delay suffers the communication between nodes. This work combines those routing metrics as a composite routing metric, i.e., measured as link cost, to calculate the weight of paths and to construct the DODAG structure. Using the improved MRHOF, the proposed methodology can provide balance on communication latency and reliability, and achieve improved performance.

\subsubsection{Improved MRHOF hysteresis function}

Using the trickle algorithm, the LB-RPL broadcasts the DIO messages, and informs the link cost metrics of the node to its neighboring nodes through the DIO messages. The path cost is a sum of the selected metrics to the path, which is advertised by the parent. The metrics are hop count, workload, and ETX, and they are used in the estimation of link. The value of rank estimated in the proposed methodology is given below.

$$
\begin{gathered}
\text { Path Cost }=\text { Parent }_{\text {pathcost }}+\text { Link Cost } \\
\text { Rank }=\text { function } \min (\text { Path Cost })
\end{gathered}
$$

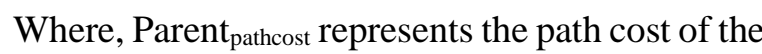
parent node, and Link cost represents the cost associated with the parents' link regarding the selected metric. The hysteresis function in MRHOFgoverns the parent selection using the Eqs. (4) and (5). Where, Np1 and Np2 are the current best parent and candidate parent node, respectively. The threshold is the hysteresis function, and it represents 
the minimum difference between the cost of the preferred and candidate parent.

$$
\begin{gathered}
\text { Preferred parent }= \\
\left\{\begin{array}{c}
N p 2 \text { if Np2 path cost }<N p 1 \text { path cost }+ \\
\text { parent switching threshhold } \\
N p 1 \text { else }
\end{array}\right. \\
\text { Linkcost }=\left(1-\frac{1}{\operatorname{Hopcount}(N)}\right) \\
+ \text { Workload }(N)+\operatorname{ETX}(N)
\end{gathered}
$$

Where, Hop count, workload, and ETX represent the number of hops to reach the root node from node N, Packet sending rate of the parent node, and ETX of the link between node $\mathrm{N}$ and parent node, respectively.

$$
E T X=E T X \_O L D \times 0.9+E T X \_N E W \times 0.1(8)
$$

Where, ETX $\mathrm{OLD}_{\text {is }}$ is the previous ETX value, and ETX $_{\text {NEW }}$ is calculated by ETX $=1 /(\mathrm{Df} * \mathrm{Dr})$. The Df and $\mathrm{Dr}$ represent the measured probability that a packet is received by the neighbor and the measured probability that the acknowledgment packet is successfully received, respectively. Once a network is initialized, all the nodes are assigned with a default value $\operatorname{ETX}(\mathrm{N})$. It facilitates the ETX of the nodes to gradually converge to the actual value after several times of update.

The main problems associated with the proposed hysteresis function are taking an optimal decision on the parent set size and parent switching threshold value. The first problem is solved by fixing the minimum and the maximum number of parent candidates. The proposed methodology decides the value of the minimum number of parent candidates as 2. The maximum parent set size is decided based on child count. To select the maximum parent set size nearer to the child count, the value of $\gamma$ is set as 0.5 and above.

$$
\begin{gathered}
\text { maxparent set size }=\gamma \times \text { child count } \\
\text { Where, the } \gamma \in[0.5,1]
\end{gathered}
$$

When a node joins in the new group due to the successful DIO transmission in the trickle algorithm, the transmission path may be better than others. It suddenly attracts many nodes and makes huge changes in the DODAG structure immediately. It makes a negative impact on network stability and affects routing performance.

\subsubsection{Optimal parent switching algorithm for network} stability

By making an optimal decision on the parentSwitch Threshold, the negative impact of network instability on the routing performance can be drastically reduced. To attain this aim, the proposed methodology takes into account the network instability due to the decision of the trickle algorithm to decide the parent switching threshold. Without considering the parent switching threshold, node A suddenly attracts more children of other neighboring nodes, such as nodes B and C, whose have slightly higher rank value than node $\mathrm{A}$, when once a node $\mathrm{A}$ announces a small rank value through DIO transmission. It impacts the network stability as well as high energy loss. The changes in the DODAG structure as per the hysteresis algorithm gives some benefits to the RPL routing. Indeed, the impact of this DODAG structure on the number of DIO transmissions and energy loss is higher than its positive impact on the packet delivery ratio.

To better understand the problem, consider a network that is depicted in the following Fig. 2. In this network, nodes $\mathrm{B}$ and $\mathrm{C}$ are two parents, and they hold some of their neighboring nodes in their DODAG structure. A common neighbor to both of them is node A. When node A has low-rank value than nodes $\mathrm{B}$ and $\mathrm{C}$, and it announces the rank value through DIO transmission. In the Fig. 2, the rectangle built with solid line show the joint coverage areas of those nodes. The left one or a rectangle with dashed line is the joint coverage area of nodes B and A, and the rectangle in the right side or rectangle with dotted line is the joint coverage area of nodes A and C. The center rectangle area represents the joint coverage area of nodes A, B, and C. If the rank of nodes B and $\mathrm{C}$ are greater than that of node $\mathrm{A}$, the node $\mathrm{A}$ starts to broadcast the DIO messages to restructure the DODAG structure and so it abruptly absorbs a significant portion of children from the nodes B and C. The node Aattracts the children, which are represented in dashed line. This sudden change tends to network instability, especially in the large-scale and high traffic network.

Due to the dynamicity in high traffic networks, a rank value of a parent candidate cannot reflect the real state of it thoroughly. The proposed algorithmexploits the parent switching threshold to take switching decision between the preferred and candidate parents, instead of considering only the node's rank value to mitigate the impact of networkinstability on control overhead and routing performance. Moreover, the proposed methodologydecides the threshold value that should 


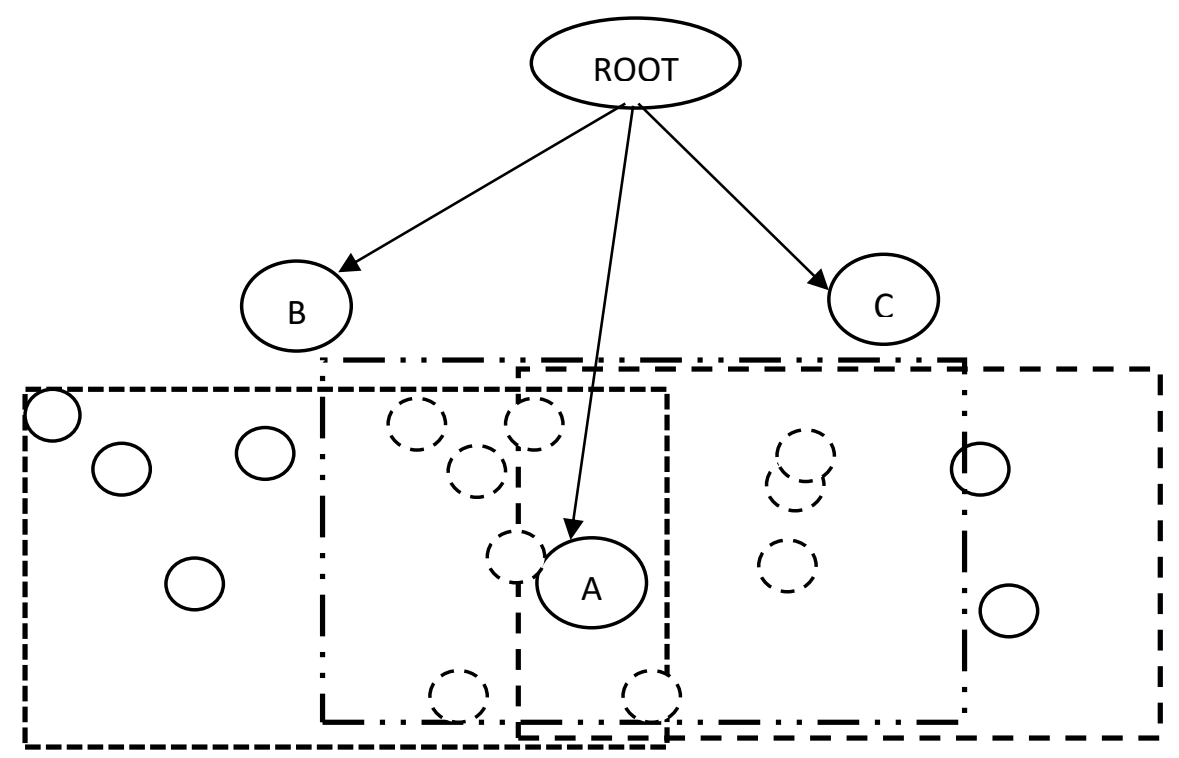

Figure. 2 Sample network for understanding network instability problem

be equal to the one minus the inverse of minimum node density or $\left(1-1 / \mathrm{k}_{\mathrm{min}}\right)$. As high node density induces high overhead due to frequent parent switching, a high value of parent switching threshold lowers the possibility of network instability, while the cost of a current parent node gradually converges to the actual value according to Eq. (6). The parent switching threshold decreases the overhead induced by the parent changes, which in turn lessen the control traffic overhead as well as mitigate the load imbalancing. Thus, the efficiency of the trickle algorithm decides the performance of improved MRHOF on the RPL routing protocol [7].

\subsection{Trickle algorithm for broadcast suppression using local-topology adaptive $k$-value}

An important parameter in the Trickle algorithm is the redundancy constant $\mathrm{k}$. However, its benefits on the efficiency of trickle are not entirely utilized. The selection of high and low $\mathrm{k}$ value without depending on the network topology tends to unnecessary DIO transmissions and suppressions, respectively, when comparing with the consistent message counter, $\mathrm{c}$. The randomly chosen parameters in message suppression of Trickle can cause some nodes to remain silent for long periods, resulting in some optimal routing paths to remain undiscovered. Thus, the proposed methodology decides the value of $\mathrm{k}$ depending on the node density in local topology using the function $\mathrm{F}(\mathrm{K})$ in Eqs. (10), (11), and (12).

$$
F(K)=\left\{\begin{array}{c}
\mathrm{k}_{\min }, \alpha C<\mathrm{k}_{\min } \\
\lfloor\alpha\rfloor, \mathrm{k}_{\min }<\alpha C>\mathrm{k}_{\max } \\
\mathrm{k}_{\text {max }}, \quad \alpha C>\mathrm{k}_{\min }
\end{array}\right.
$$

Where, $\alpha \in[0.5,1]$

$$
\begin{gathered}
k_{\text {max }}=|N| \times \pi R^{2} / \text { Area } \\
k_{\text {min }}=\beta \times k_{\text {max }} \\
\text { Where, } \beta \in[0,0.5]
\end{gathered}
$$

The maximum and minimum value of consistent message redundancy threshold, $\mathrm{k}_{\max }$ and $\mathrm{k}_{\min }$ are decided based on node density, which is evaluated in the Eqs. (11) and (12), respectively. The function of $\mathrm{F}(\mathrm{K})$ returns the value for variable $\mathrm{k}$. The control parameters $\alpha$ and $\beta$ values are decided. Allowing very low value to $\alpha$ may tend to very low $F(K)$ and unnecessary DIO transmissions when meeting the second condition in the Eq. (7). In contrast, the high value of $\beta$ tends to unnecessary DIO suppressions, since the selection of large $\mathrm{k}$ value with high node density. Thus, the proposed methodology decides the $\beta$ value within 0 to 0.5. In the Eq. (11), CR and Area represents the communication range of a node in the network and size of the network respectively. The Eq. (11) returns the value of node density for the whole network area.

\subsubsection{Successive DIOs suppression-counter adaptive $t$ selection}

Another main problem associated with the trickle algorithm is the proper selection of $t$ value. At the end of the current interval, the redundancy counter $\mathrm{c}$ is greater than or equal to the redundancy constant, $\mathrm{k}$, the Trickle enables a node to suppress its scheduled message. Otherwise, a node transmits the message. After taking the suppression decision, the redundancy counter $\mathrm{c}$ value is set to zero. However, DIOs 
transmissions from $t$ to the end of the interval are not utilized when using the randomly chosen $t$ value, and it tends to more power and energy consumption [22]. Moreover, a node in Trickle believes that it selects an optimal transmission time, $t$ value and it is sufficient to hear enough number of DIOs transmissions from its neighboring nodes. However, the randomly chosen $\mathrm{t}$ value has less possibility to satisfy the condition above always. To avoid such load imbalancing issue, the proposed methodology takes into account the successive DIOs suppressions as a variable s.

The proposed successive DIO suppression count based Trickle algorithm exploits the following three parameters:

\section{$I_{\min }$-Minimum interval length \\ $I_{\max }$-Maximum interval length \\ $\mathrm{k}$-Redundancy constant}

The proposed Trickle algorithm exploits the following three variables:

$I$ - Current interval length between $\left[I_{\min }, I_{\max }\right]$.

$\mathrm{c}-$ Redundancy counter which represents the number of consistent DIO messages in I

$\mathrm{t}-\mathrm{A}$ random time in $\left[\left(0, \mathrm{I} / 2^{(\mathrm{s}+1)}\right)\right] \mathrm{t}-\mathrm{A}$ randomly chosen transmission time in $\left[\left(0, \mathrm{I} / 2^{(\mathrm{s}+1)}\right)\right]$

Most of the conventional schemes allow the nodes to increment the value of $\mathrm{c}$ when they receive consistent messages from its neighboring nodes. However, there is a possibility of losing the transmitted consistent messages from its neighboring nodes due to network traffic. The improper value of c tends to unnecessary DIO transmissions in the network. To ensure the proper c value measurement, the protocol uses a CVALUEINC parameter, which defines the smallest permitted increase in c per node over $\mathrm{t}$ time. As a result, the node's $\mathrm{c}$ value is equal or closer to the value of the real one. The value of CVALUEINC is decided based on its packet loss ratio of a node $\mathrm{Pt}$, due to network collision. The algorithm is explained as follows.

Function 1: In the Initialization step, the proposed trickle algorithm selects an interval in-between $\mathrm{I}_{\min }$ and $\mathrm{I}_{\max }$.

Function 2: If it is a first and second interval after the network initialization, the proposed methodology decides to set the value of $I$ is equal to $I / 2$. The reason behind the idea is to avoid high control overhead induced by the nodes to converge quickly when a node selects low $I_{\min }$ value initially. The redundancy counter $\mathrm{c}$ is reset as zero. From the third interval, this phase selects a random time $t$ in $\left(0, I / 2^{(s+1)}\right)$ instead of choosing it in $[I / 2, I]$ like in the basic Trickle algorithm. Moreover, it also initializes a variable $\mathrm{c}$ to zero. The reasons behind the idea of selecting $t$ value in $\left(0, \mathrm{I} / 2^{(s+1)}\right)$ is the selection of $t$ imposes a delay of at least $\mathrm{I} / 2$ before attempting to resolve the inconsistency problem, and it accumulates the delay in every hop in the routing path. Where variable $s$ represents the number of successive DIO suppressions. Moreover, some nodes have more chances to transmit than others, when it selects t from $(0, \mathrm{I})$.

\section{Proposed trickle algorithm}

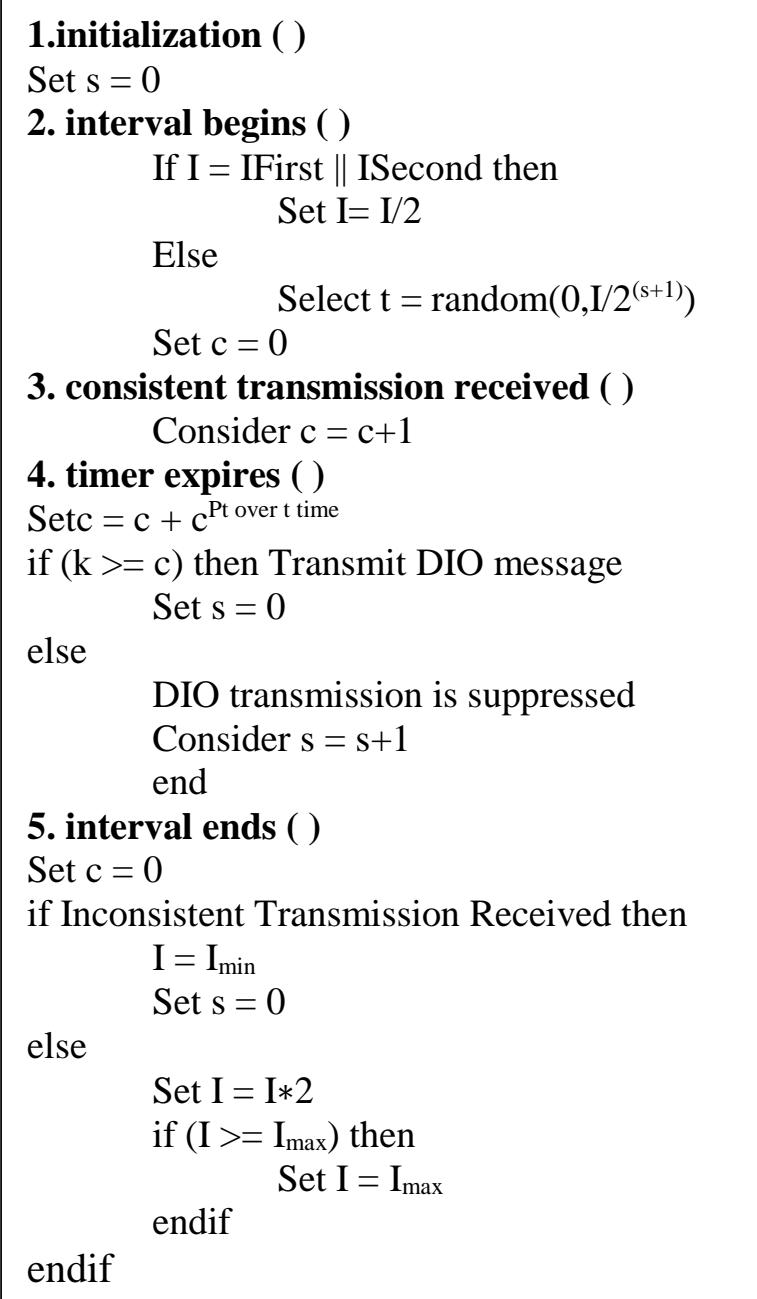

Function 3: If consistent transmission is received, the value of $\mathrm{c}$ is increased by one.

Function 4: The fourth function starts to run when the timer $t$ is expired. It starts to compare the value of redundancy constant $\mathrm{k}$ and redundancy counter $\mathrm{c}$. If the value of $\mathrm{c}$ is less than or equal to the value of $\mathrm{k}$, then it simply transmits its DIO message, and the value of variable $\mathrm{s}$ is set to zero. But if the value of variable $\mathrm{c}$ is larger than the value of redundancy constant $\mathrm{k}$, then the DIO transmission is suppressed, and the $\mathrm{s}$ variable is incremented by 1 . A node with high $\mathrm{s}$ value whose number of successive DIO suppressions are high obtains more chance to send its 
DIO message in next turn because the random time $t$ is indirectly proportional to the value $\mathrm{s}$.

Function 5: When the interval end () function is executed, the value of $c$ is reset. If no inconsistent messages are heard, the trickle enforces the nodes to double the length of current interval simply. Otherwise, it simply sets $I$ to $I_{\min }$ and also sets the variable s to zero. If the value of I exceeds the value of $I_{\max }$, it resets I to Imax only.

The pseudocode of the new algorithm is presented in the above algorithm. On the basis of the number of DIOs suppressions, the proposed LoBRPL decides DIO broadcasting effectively. Moreover, assigning a proper value to the trickle variables $\mathrm{c}, \mathrm{k}$, and parent switching threshold improves the performance of the Trickle algorithm and MRHOF in RPL. It results in balanced energy consumption, reliable data forwarding in the network layer, as well as efficient data aggregation in the application layer over IoT.

\section{Performance evaluation}

To assess the proposed work LoB-RPL, the Cooja simulator running on the Contiki Operating System is used. The Cooja simulator is an open-source simulator/emulator, and it simulates the IoT applications. Moreover, the implementation of the basic RPL in this simulator is easy, and any improvement in the RPL functions, such as MRHOF and trickle algorithm, is flexible in the Cooja simulator. Table 1 describes the different parameters used for performance evaluation.

\subsection{Performance metrics and evaluation scenarios}

The performance comparison is made between the proposed LoB-RPL and the MRHOF RPL [3].To show the performance improvement of the proposed work, it is compared with the RPL. In comparative work, RPL includes two main components, such as MRHOF and new dynamic Trickle algorithm [20]. The RPL constructs the tree, called DODAG using those components, and executes the many-to-one traffic. The MRHOF specifies how routing metric is computed and used during topology construction. The MRHOF routing metric in the existing RPL is hop count, because of showing the performance improvement of LoB-RPL exactly due to the considered composite metrics, such as ETX and workload. Another reason to usethe hop count in the existing MRHOF is that it is a main reason to create the hot spot around rootnode and worsens the routing performance under high traffic scenario, though it is
Table 1. Simulation parameters

\begin{tabular}{|l|c|}
\hline \multicolumn{1}{|c|}{ PARAMETER } & VALUES \\
\hline Simulator & Cooja Simulator \\
\hline Number of nodes & Sky Mote \\
\hline Sensor type & Constant Bit Rate \\
\hline Traffic type & 200m x 200m \\
\hline Area & 50m \\
\hline Communication range & ContikiMAC, IPv6 \\
\hline MAC type & UDG Model \\
\hline Propagation type & RPL \\
\hline Routing protocol & UDP \\
\hline Transport agent & seconds \\
\hline $\begin{array}{l}\text { Inter packet arrival } \\
\text { time(sec) }\end{array}$ & 180 seconds \\
\hline Simulation time & \\
\hline
\end{tabular}

a good routing metric. Using the hysteresis mechanism, the MRHOF in RPL selects a candidate parents the preferred parent, when it has a better rank value than the rank of current preferred parent minus from threshold.

Moreover, the dynamic trickle algorithm applies neighbour count based transmission time selection. However, the selection of transmission time imposes a delay of at least $\mathrm{I} / 2$ and it accumulates the delay in every hop towards the gateway. Thus, the RPL with MRHOF and dynamic trickle algorithm is more suitable to show the importance of proposed LoBRPL in simulation.

The comparison is done using the routing metrics, such as packet delivery ratio, packet loss, throughput, control overhead, avg hop count, and expected transmission count. The metrics are evaluated under the scenario of varying inter-packet arrival time from 10 to $40 \mathrm{sec}$, i.e., data transmission interval.

Packet Delivery Ratio: The ratio of delivered data packets to the total number of transmitted data packets.

Packet Loss: Total numbers of lost packets before reaching the root node.

Throughput: It refers that how much amount of data in bytes is delivered to the root node per second.

Control Overhead: The numbers of control packets involved in the DODAG construction as well as in the data transmission. 


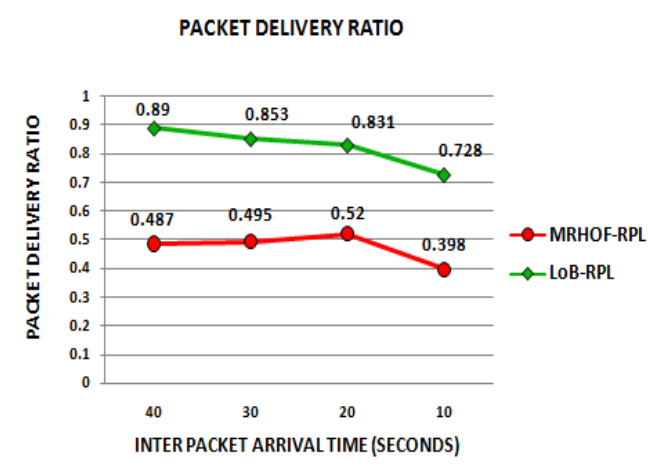

(a)

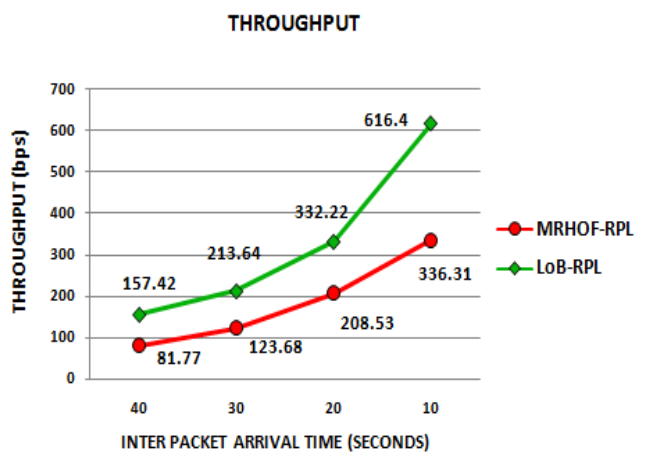

(c)

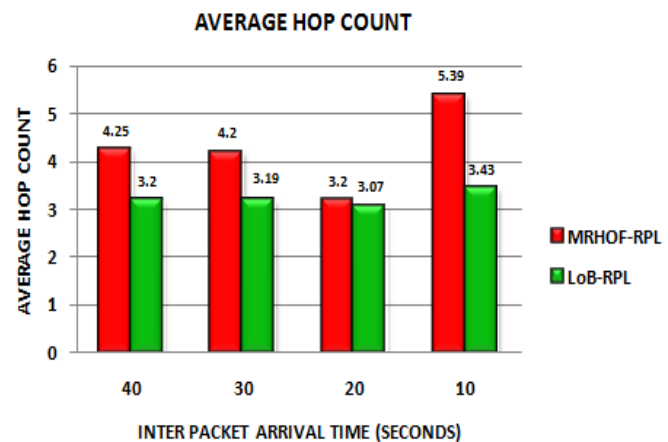

(e)

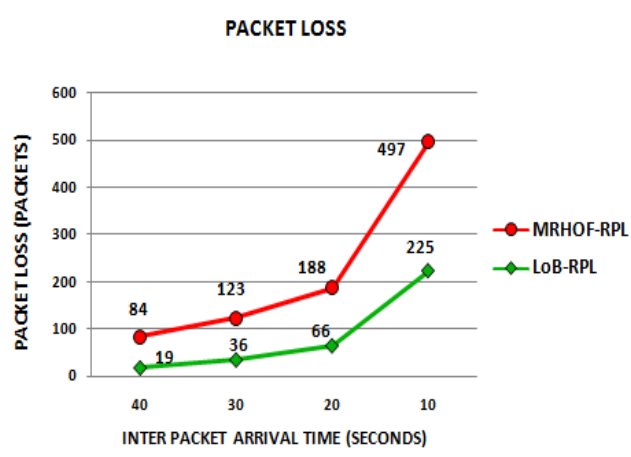

(b)

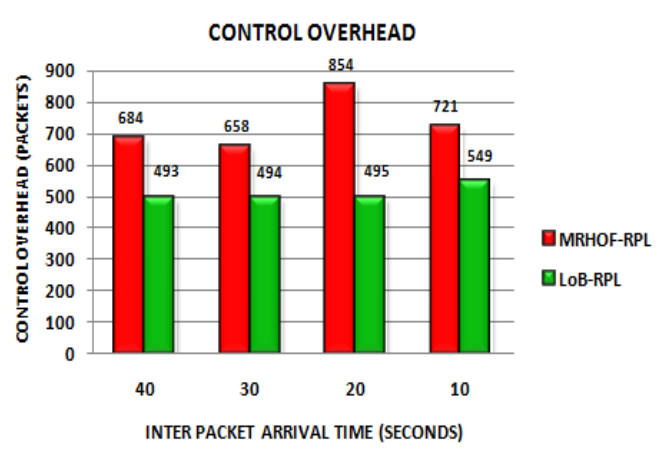

(d)

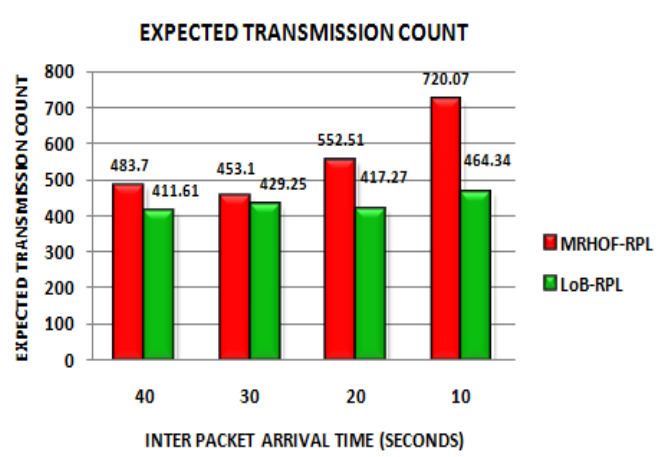

(f)

Figure. 3 Comparison graph with proposed LoB-RPL with existing MRHOF RPL:

(a) number of nodes vs. packet delivery ratio, (b) number of nodes vs. packet loss, (c) number of nodes vs. throughput,

(d) number of nodes vs. controloverhead, (e) inter packet arrival time vs. average hop count, and

(f) inter packet arrival time vs. expected transmission count

Average Hop Count: Average numbers of routers are involved in the DODAG structure for data forwarding.

Expected Transmission Count: The expected numbers of transmissions are involved in delivering the data packets to the root node on average.

\subsection{Simulation results}

This section demonstrates the simulation results by varying the inter-packet arrival time.

\section{Packet delivery ratio and packet loss}

From Fig. 3 (a) and (b), it is observed that the packet loss problem even becomes more serious when the inter-packet arrival time downs or the network traffic increases. In the case of highnetwork traffic, RPL experiences up to $40 \%$ of packet delivery ratio, while that of LoB-RPL is above $72 \%$. In Fig. 3 (a), the percentage of packet delivery ratio in the proposed work is more than $70 \%$ in all five network traffic scenarios. Because the consideration of workload in the proposed work ensures the parent change in DODAG structure and the incorporation of parent switching threshold avoids infrequent parent 
change as well as network instability. As only the rank value of a parent candidate cannot reflect the real state of it thoroughly, the parent switching threshold is used to take switching decision between the preferred and candidate parents and improve the performance of the proposed work. The percentage of packet delivery ratio in the LoB-RPL is much higher than that of MRHOF RPL with MRHOF, even for a large and small inter-packet arrival time. By fixing the minimum and maximum number of parent candidates, the LoB-RPL limits the size of DODAG structure and avoids unnecessary packet loss in the IoT environment. From Fig. 3 (c) the difference of packet loss between the proposed and existing works is approximately more than 70 packets in all scenarios. The LoB-RPL routinely changes the DIOs transmitters and packet loss. For instance, the MRHOF RPL experiences 84 lost packets, whereas the proposed works lose only 19 packets in low traffic scenarios.As per the existing work, if a node with slightly high rank compared to the current parent node may suddenly attracts more number of children of other neighbouring nodes, it makes changes in the DODAG structure and it affects the packet delivery ratio.Moreover, the neighbor count based DIO transmission selection in existing MRHOF-RPL tends to improper decision, when the network traffic is high.

\section{Throughput and controloverhead}

Fig. 3 (c) and (d) demonstrate the result of throughput and control overhead for both the proposed LoB-RPL and MRHOF RPL.

When the network has high traffic, the LoB-RPL provides better throughput than MRHOF RPL. As the number of hops is not only the metric on the efficiency of communication, LoB-RPL can find the best path with minimum hops and high link quality routers quickly rather than using ETX. When the network traffic increased, the difference in throughput between LoB-RPL and MRHOF RPL is higher than in the less traffic scenario or when the inter-packet arrival time is high. Because, the MRHOF-RPL tends to hotspot problem, when the nodes forward most of the data packets through the routers, nearer to the root node. It is because, dynamic DIO transmission time selection irrespective of the DIO suppression in previous interval tends to unnecessary delay or DIO packet loss. This increases the delay of packets. It results in poor throughput in MRHOF-RPL. For instance, the MRHOF-RPL attains 80 bps throughput, when the inter-packet arrival time is $40 \mathrm{sec}$, whereas the LoB-RPL attains $150 \mathrm{bps}$ at the same scenario. The proposed work supports dense scenario also, since it decides the threshold value i.e. one minus the inverse of minimum node density. Because, high node density induces high overhead due to the frequent parent switching, high value of parent switching threshold lowers the possibility of network instability. Afterthat, the cost of a current parent node will gradually converges to the actual value. Thus, the parent switching threshold in LoB-RPL decreases the overhead induced by the parent changes, which in turn lessen the control traffic overhead as well as improve the network throughput. For instance, the MRHOF-RPL attains 336.31 bps throughput, when the inter-packet arrival time is $10 \mathrm{sec}$, whereas the LoB-RPL attains $616.4 \mathrm{bps}$ at the same scenario.

Fig. 3 (d) shows that the standard RPL with MRHOF provides a high value of control overhead due to the use of the ETX metric. The MRHOF based on ETX enables the nodes to transmit more packets to obtain a successful parent node among neighboring nodes. As the RPL advertises the routing metrics to its neighbouring nodes by broadcasting the RPLDIO messages and it is optimized using the trickle algorithm. A main reason behind that the standard RPL provides more control traffic is basic Trickle algorithm, due to the improper selection of consistent message transmission counter and threshold. With the inter-packet arrival time of $40 \mathrm{sec}$, the LoB-RPL utilizes only 490 packets, whereas MRHOF RPL uses 690 packets in the same scenario. However, in the low dense network, both the works show similar performance. That is, the impact of the high value of $t$ on routing overhead is relatively small, and so, it does not cause too much difference with LoB-RPL. However, the same $t$ value for other scenarios tends to the selection of poor quality parent nodes due to improper DIO message broadcast suppression/transmission.It is because, the MRHOFRPL selects the value of $t$ only based on the neighbor count, but not the traffic rate.The compensation for random selection of $t$ in LoB-RPL is the consideration of successive DIOs suppression counter, and this makes the protocol offer more chances for DIOs transmission to nodes, which suppresses DIOs highly in the past. This reduces unnecessary DIOs transmission and control overhead. At the inter-packet arrival time of $20 \mathrm{sec}$, the control overhead of LoB-RPL is only 495 packets, whereas MRHOF RPL uses 854 packets in the same scenario.

\section{Average hop count and expected transmission count}

Fig. 3 (e) and (f) show the result of average hop count and expected transmission count for LoBRPLand MRHOF RPL under various inter-packet arrival times. It shows that the result of the proposed work is always better than MRHOF RPL.For instance, 
the hop count of the proposed work is 3.45 , but the existing work reaches 5.39 hops on average. The restriction over unnecessary DIOs transmission through the usage of the CVALUEINC parameter and the permission for the smallest increase in c per node over $t$ time ensures an effective DODAG creation as well as maintenance. It enables all the nodes to spend their energy evenly and avoids the hotspot problem around the root node. Instead of providing more chances for data transmission to some nodes, the proposed work splits the load among various nodes by selecting the $t$ value from $(I / 2, I)$ it balances the hop count as well as expected transmission count in the proposed work. The difference of the expected transmission count between the proposed and existing work is nearly 260 transmissions when the inter-packet arrival time is 10 sec. A main reason behind this is the improper value of $\mathrm{c}$ which tends to unnecessary DIO transmissions and data loss in the network. To ensure the proper $\mathrm{c}$ value measurement and avoids unnecessary increment in ETX, the LoB-RPL protocol uses a CVALUEINC parameter, which is decided on the basis of own packet loss ratio of a node. It reduces the ETX in the proposed work and improves the LoBRPL routing performance.

\section{Conclusion}

This paper presented an energy-efficient routing in the network layer of IoT for avoiding hotspot creation and ensuring an efficient data aggregation in the application layer. To attain the aim, the proposed LoB-RPL has incorporated the composite metric based parent selection, DODAG construction, and local topology adaptive decision on trickle parameters. The usage of ETX, Workload, as well as hop count value, effectively balances the routing performance and energy consumption. The consideration of workload assists the MRHOF to offer a load-balanced data transmission in RPL and avoid hotspot creation nearer to the root node. By tuning the parameters of the Trickle algorithm based on node density, unnecessary DIO transmissions and suppressions are prevented. Although there is a possibility for frequent parent switching in RPL, due to the consideration of dynamic parameters, the LoBRPL avoids frequent changes in DODAG structure by deciding the parent switching threshold. From the simulation results, it is observed that the performance of the proposed work is improved than the MRHOF RPL in all the metrics. The proposed LoB-RPL increases the throughput from 157.4 to 616.4 bps when the inter-packet arrival time is decreased from 40 to $10 \mathrm{sec}$. However, the existing work RPL increases the throughput from 81.7 to $336.3 \mathrm{bps}$ under the same scenario. An interesting future direction is to shift the attention from single layer to multi layer for achieving an efficient data aggregation in IoT. Moreover, incorporating node capacity related metrics in OFs will further improve the performance of RPL as well as data aggregation efficiency under IoT environment.

\section{Conflicts of Interest}

"The authors declare no conflict of interest."

\section{Author Contributions}

"Conceptualization, Vanathi Arunachalam and Nagamalleswara Rao Nallamothu; methodology, Vanathi Arunachalam; software, Vanathi Arunachalam; validation, Vanathi Arunachalam; formal analysis, Vanathi Arunachalam; investigation, Vanathi Arunachalam; resources, Vanathi Arunachalam; data curation, Vanathi Arunachalam and Nagamalleswara Rao Nallamothu; writingoriginal draft preparation, Vanathi Arunachalam; writing - review and editing, Vanathi Arunachalam and Nagamalleswara Rao Nallamothu; visualization, Vanathi Arunachalam and Nagamalleswara Rao Nallamothu; supervision, Nagamalleswara Rao Nallamothu; project administration, Nagamalleswara Rao Nallamothu;".

\section{References}

[1] I. Yaqoob, E. Ahmed, I. A. T. Hashem, A. I. A Ahmed, A. Gani, M. Imran, and M. Guizani, "Internet of Things Architecture: Recent Advances, Taxonomy, Requirements, and Open Challenges", IEEE Wireless Communications, Vol. 24, No. 3, pp. 10-16, 2017.

[2] J. V. V. Sobral, J. J. P. C. Rodrigues, R. AL. Rabêlo, J. Al-Muhtadi, and V. Korotaev, "Routing protocols for low power and lossy networks in internet of things applications", Sensors, Vol. 19, No. 9, pp. 2144, 2019.

[3] T. Winter, P. Thubert, A. Brandt, J. Hui, R. Kelsey, P. Levis, K. Pister, R. Struik, JP. Vasseur, and R. Alexander, RPL: IPv6 Routing Protocol for Low-Power and Lossy Networks, Vol. 6550, RFC, 2012.

[4] J. Marietta and B. Chandra Mohan, "A Review on Routing in Internet of Things", Wireless Personal Communications, Vol. 111, pp. 209233, 2020.

[5] H. Kharrufa, H. AA. Al-Kashoash, and A. H. Kemp, "RPL-based routing protocols in IoT 
applications: A Review", IEEE Sensors Journal, Vol. 19, No. 1 pp. 5, 5952-5967, 2019.

[6] H. Lamaazi and N. Benamar, "A comprehensive survey on enhancements and limitations of the RPL protocol: A focus on the objective function", Ad Hoc Networks, Vol. 96, 2020.

[7] P. Levis, T. Clausen, J. Hui, O. Gnawali, and J. Ko, The Trickle Algorithm, Internet RFC 6206, 2011.

[8] P. O. Kamgueu, E. Nataf, and T. D. Ndie, "Survey on RPL enhancements: a focus on topology, security and mobility", Computer Communications, Vol. 120, pp. 10-21, 2018.

[9] S. A. Dehkordi, K. Farajzadeh, J. Rezazadeh, R. Farahbakhsh, K. Sandrasegaran, and M. A. Dehkordi, "A survey on data aggregation techniques in IoT sensor networks", Wireless Networks, Vol. 26, No. 2, pp. 1243-1263, 2020.

[10] S. Sennan, S. Balasubramaniyam, A. Kr. Luhach, S. Ramasubbareddy, N. Chilamkurti, and Y. Nam, "Energy and Delay Aware Data Aggregation in Routing Protocol for Internet of Things", Sensors, Vol. 19, No. 24, pp. 5486, 2019.

[11] B. Ghaleb, A.Y. Al-Dubai, E. Ekonomou, A. Alsarhan, Y. Nasser, L.M. Mackenzie, and A. Boukerche, "A Survey of Limitations and Enhancements of the IPv6 Routing Protocol for Low-Power and Lossy Networks: A Focus on Core Operations", IEEE Communications Surveys \& Tutorials, Vol. 21, No. 2, pp. 1607$1635,2019$.

[12] B. Djamaa and M. A. Richardson, The trickle algorithm: issues and solutions, Research Report, 2015.

[13] T. Zhang and X. Li, "Evaluating and Analyzing the Performance of RPL in Contiki", In: Proc. of the First International Workshop on Mobile Sensing, Computing and Communication, pp 19-24, 2014.

[14] P. O. Kamgueu, E. Nataf, T. D. Ndié, and O. Festor, Energy-based routing metric for RPL, Research Report, RR-8208, HAL, 2013.

[15] A. Barbato, M. Barrano, A. Capone, N. Figiani, "Resource oriented and energy efficient routing protocol for IPv6 wireless sensor networks", In: Proc. of IEEE Online Conf. on Green Communications (OnlineGreenComm), pp. 163-168, 2013.

[16] M.Zhao, P. H. J. Chong, H. C. B. Chan, "An energy-efficient and cluster-parent based RPL with power-level refinement for low-power and lossy networks", Computer Communications, Vol. 104, pp. 17-33, 2017.
[17] L. Nguyen and C. Kim, "Towards skewness and balancing of RPL trees for the internet of things", CoRR, Vol. abs/1903.01839, 2019.

[18] H. Lamaazi and N. Benamar. "A novel approach for RPL assessment based on the objective function and trickle optimizations", Wireless Communications and Mobile Computing, 2019.

[19] B. Ghaleb, A. Al-Dubai, E. Ekonomou, B. Paechter, and M. Qasem. "Trickle-plus: Elastic trickle algorithm for low-power networks and Internet of Things", In: Proc. of IEEE Wireless Communications and Networking Conf. Workshops (WCNCW), pp. 103-108, 2016.

[20] M.B. Yassein, S. Aljawarneh, E. Masa'deh, B. Ghaleb, and R. Masa'deh, "A New Dynamic Trickle Algorithm for Low Power and Lossy Networks", In Proc. of International Conf. on Engineering and MIS (ICEMIS), pp. 1-6, 2016.

[21] M.B. Yassein, S. Aljawarneh, and E. Masa'deh, "A new elastic trickle timer algorithm for Internet of Things", Journal of Network and Computer Applications, Vol. 89, pp. 38-47, 2017.

[22] S. Goyal, and T. Chand, "Improved Trickle Algorithm for Routing Protocol for Low Power and Lossy Networks", IEEE Sensors Journal, Vol. 18, No. 5, pp. 2178-2183, 2018. 